\title{
The Sub-health Status of Different Social Roles and the Analysis of its Influencing Factors during the 2019 Coronavirus Disease Pandemic
}

\author{
Chen Ze Tao', Qiang Liu', WanXian Lu',*, YanBin Pan ${ }^{1}$, MiaoHang Shan ${ }^{1}$
}

Chen Ze Tao', Qiang Liu', WanXian $\mathrm{Lu}^{2, *}$, YanBin Pan ${ }^{1}$, MiaoHang Shan ${ }^{1}$ ${ }^{1}$ Nurse of Intensive Care Unit, the First Affiliated Hospital of Jinan University, Tianhe, Guangzhou, Guangdong Province, CHINA.

${ }^{2}$ Supervisor of Nursing Care, the First Affiliated Hospital of Jinan University, Tianhe, Guangzhou, Guangdong Province, CHINA.

"Chen Ze Tao and Qiang Liu are co-first authors.

The first contributors to this article are Chen Zetao and Liu Qiang

\section{Correspondence}

Ms. WanXian Lu

Supervisor of Nursing Care, the First Affiliated Hospital of Jinan University, 613 Huangpu Avenue, Tianhe District, Guangzhou City, Guangdong Province, CHINA.

Mobile no: +86 020-38688319

Email: zzyxk20@yeah.net

History

- Submission Date: 08-06-2020

- Revised Date: 08-07-2020

- Accepted Date: 25-08-2020

DOI : 10.5530/ijmedph.2020.3.29

Article Available online

http://www.ijmedph.org/v10/i3

\section{Copyright}

(C) 2020 Phcog.Net. This is an openaccess article distributed under the terms of the Creative Commons Attribution 4.0 International license.

\begin{abstract}
Aim: This paper aims at investigating the sub-health status (SHS) of different social roles during the 2019 coronavirus disease (COVID-19) pandemic and analyzing its influencing factors. Design: A cross-sectional, convenience sampling including 1062 Chinese was conducted in March 2020. Statistical analysis was then performed for the data using SPSS 22.0. Results: The total SHS score for people with different social roles during the COVID-19 pandemic was $72.808 \pm 12.034$; with the physical, psychological and social adaptive score were $80.252 \pm 12.336$, $68.753 \pm 13.281$ and $66.632 \pm 18.604$ respectively. Univariate analysis showed that SHS scores for social adaptability were different in different places of residence, as well as physical, psychological and total SHS scores for different social roles $(P<0.05)$. The multivariate analysis showed that the social roles were a factor influencing the physical SHS score $(P<0.05)$, while gender was the factor influencing the psychological SHS score $(P<0.05)$. Conclusion: Human resource management needs improvement; meanwhile health consultation, enough time to rest and health care service are essential for medical staff, Centers for Disease Control (CDC) personnel, as well as couriers. Support to maintain work-family balance is seriously expected for female employees. Overall, the top priority in controlling COVID-19 is to protect their health both mentally and physically.
\end{abstract}

Key words: The coronavirus disease, Different social roles, Sub-health status, Influencing factors.

\section{INTRODUCTION}

All age groups are susceptible to pneumonia caused by the severe acute respiratory syndrome coronavirus 2(SARS-CoV-2) novel coronavirus, known as the coronavirus disease 2019 (COVID-19) and infected cases are mainly associated with the symptoms of viral pneumonia. ${ }^{1}$ During the COVID-19 pandemic, the Chinese government has imposed control measures with a temporary shutdown of some service-oriented businesses to reduce the presence of clusters and thus lower the risk of virus transmission. To ensure the essential operations of the society and necessities of living, pandemic preventing staff in many industries have to provide public servicesat risk of exposure to the virus, for example in hospitals, CDC, public transportation, express industry and community service center. They must perform the functions of their social roles out of work responsibility and social responsibility which is the process of fulfilling social roles. ${ }^{2}$ Working under an unexpected threat from the virus, their health status can be inevitably impacted at physical, mental and social levels to some extent, thus concern over the health status of these groups is urgently needed. ${ }^{3}$

Sub-health status (SHS) is considered as a state between good health and illness characterized by

some clinical manifestations that cannot be diagnosed with any disease of clinical standards. It is also recognized as somatoform disorders and medically unexplained symptoms. ${ }^{4}$ While SHS can develop into a disease, people suffering from it may also fully recover. Furthermore, effective interventions can certainly help to facilitate recovery. ${ }^{5}$ According to a global survey by $\mathrm{WHO}$, only $5 \%$ of the world's population is truly healthy, diseases are identified in only $20 \%$ and the remaining $75 \%$ are suffering from SHS. ${ }^{6}$ Multiple studies in China have shown that the prevalence of SHS in the general population ranges from $36.23 \%$ to $68.4 \%$ and various possible symptoms and risk factors associated with SHS have been investigated and discussed. ${ }^{7-13}$ However, no related literature has reported the correlation regarding the sub-health status of people playing their different parts in the disease prevention and control under a massive pandemic. A concern is there by raised that SHS may lead to higher morbidity or cause greater health consequences due to the impact of apandemic. As such, a questionnaire-based study was conducted to investigate the status quo of sub-health during the fight against COVID-19 among the people with different social roles, to provide a theoretical basis for
Cite this article: Chen Z, Liu Q, Lu W, Pan Y, Shan M. The Sub-health Status of Different Social Roles and the Analysis of its Influencing Factors during the 2019 Coronavirus Disease Pandemic. Int J Med Public Health. 2020;10(3):135-40. 
the health sector in the development of healthcare interventions. Given that COVID-19 cases have been reported in many countries across the world with varying prevalence, ${ }^{14}$ this study may also serve as a report from which the pandemic prevention staff in other countries or regions may learn from to proactively respond to the spread of COVID-19.

\section{MATERIALS AND METHODS}

\section{Subjects}

Convenience sampling was used to select the COVID-19 pandemic preventing staff as the study subjects. Inclusion criteria: 1) subjects who provided informed consents and volunteered to participate in this investigation; 2) people working during the pandemic in China, including medical staff, CDC personnel, scientific researchers, government officials, community service workers, public transport employees, police, couriers, volunteers, as well as staff in other positions. This study has been approved by the medical ethics committee of the first affiliated hospital of Jinan University (approval number: KY-2020-016).

\section{Research Methods \\ Research Tools}

\section{General information questionnaire}

Survey form for general information was designed by the investigator and consisted of 17 survey items, including Gender, age, height, weight, nationality, place of origin, current residence, family residence, education, marital status, only child, political status, occupation, social role, monthly salary, virus exposure history and family infection history.

\section{SHS questionnaire of Chinese residents during COVID - 19 pandemic}

This questionnaire was designed based on the sub-health rating scale (SHMSV1.0) developed by Xu Jun. ${ }^{13}$ This modified scale would contain three dimensions making up 3 subscales, namely physical sub-health (1-14 items), psychological sub-health (16-27 items) and social adaptive sub-health (29-37 items) with a total of 39 items. The items 15, 28, 38 and 39 were used for the overall evaluation of the health status within each dimension and would not be taken into account for the score rating. A commonly used 5-point Likert scale was developed to incorporate the positive items, including questions 1-3, 13-19 and 26-39 with a scaled score equal to the raw score at $1-5$; while negative items including questions 4-12 and 20-25 with a scaled score equal to 6 min the raw score. For each subscale, the sum of all item scores was the raw score. The total raw score was calculated as the sum of raw scores derived from all 3 sub-scales. For any individual dimension, a lower score indicated higher severity of SHS. For sub-scales and the whole scale, raw scores were converted into percentile scores for statistical analysis. Converted score $=($ raw score - minimum theoretical score $) /($ maximum theoretical score - minimum theoretical score $) \times 100$. The highest and lowest converted scores of the three subscales were 0 and 100 respectively. In this study, analysis of sub-health was performed using converted scores for all subscales and the whole scale. Based on the sub-health rating scale developed by Xu Jun, the health condition of subjects would be assessed as disease status (scored $<54$ ), SHS (scored 54 to 79) and health status (scored > 79). ${ }^{13}$ This questionnaire exhibited a content validity index Kaiser-Meyer-Olkin (KMO) of 0.859 and a Cronbach's $\alpha$ coefficient of 0.912 , indicating good reliability and validity.

\section{Data Collection}

Data were collected throughWenjuanxing (wjx.cn; an online platform available for questionnaire sources) from March 6 to 9, 2020 and all the information should be completed before a questionnaire could be submitted. To ensure the acceptance of those questionnaires, 1) invalid questionnaires which were completed in less than $2 \mathrm{~min}$, as well as where the same answers were found for all options should be excluded from the study; 2) only the first round of answers submitted were considered valid by screening per IP addresses and time of response to prevent multiple questionnaires exported from one single machine; 3) staff who were not working or were preparing to participate in the battle against COVID-19. A total of 1088 questionnaires were dispensed and 1088 were collected. By excluding the 18 questionnaires that were completed in less than $2 \mathrm{~min}$ and the 8 questionnaires completed improperly, 1062 eligible questionnaires were eventually acquired, showing an effective recovery rate of $97.6 \%$.

\section{Data Analysis}

Data analysis was performed using SPSS22.0. Descriptive statistics included frequency, proportion and mean $\pm \mathrm{SD}$. The comparison of general data between groups was performed by $t$-test and the comparison between multiple groups was performed by One-way analysis of variance ANOVA. Multiple linear regression was used to analyze the influencing factors among gender, political status, residence and social roles, using a two-sided test at $\alpha=0.05 P<0.05$ was considered statistically significant.

\section{RESULTS}

\section{General Demographic Characteristics}

In this study, a total of 1062 pandemic preventing staff fighting the COVID-19 were investigated, including 572 males (53.9\%) and aged 17 to 67 years with a mean age of $(24.929 \pm 9.009)$ years. The rest of the general information is shown in Table 1.

\section{Health Condition of People with Different Social Roles during the COVID-19 Pandemic}

Of the 1062 staff against COVID-19 included in this study, 61 were assessed to have disease status (5.7\%), 659 SHS (62.1\%) and 342 health status (32.2\%). The subjects who were assessed to have SHS in different social roles, including couriers, volunteers, medical staff, staff in other positions, community service workers and public transport employees accounted for $66.6 \%, 63.37 \%, 63.16 \%, 63.02 \%, 57.53 \%$ and $52.94 \%$. By place of residence, $62.9 \%$ of urban residents and $61.7 \%$ of rural residents were assessed to have social adaptive SHS.

In this study, the total sub-health score for the pandemic preventing staff was $78.808 \pm 12.034$, with a mean score of $2.080 \pm 0.344$ based on all items. Among the three subscales, the highest score $80.252 \pm 12.336$ was noted in physical sub-health status, followed by $68.753 \pm 13.281$ for psychological sub-health status and $66.632 \pm 18.604$ for social adaptive sub-health status. (Table 1). Sequentially, lower physical sub-health status scores were observed as $64.120 \pm 12.247$ for couriers, $67.142 \pm 13.627$ for CDC personnel and $70.031 \pm 12.731$ for medical staff among the various social roles.

\section{Univariate Analysis of SHS and General Information for People with Different Social Roles during the COVID-19 Pandemic}

Based on the univariate analysis, significant differences can be found in the physical sub-health status scores for pandemic preventing staff holding a diverse range of social roles $(P<0.05)$; differences were also observed in psychological sub-health status scores as it pertains to gender, social roles $(P<0.05)$. Moreover, differences were again observed in social adaptive sub-health status scores by place of residence $(P<0.05)$; 


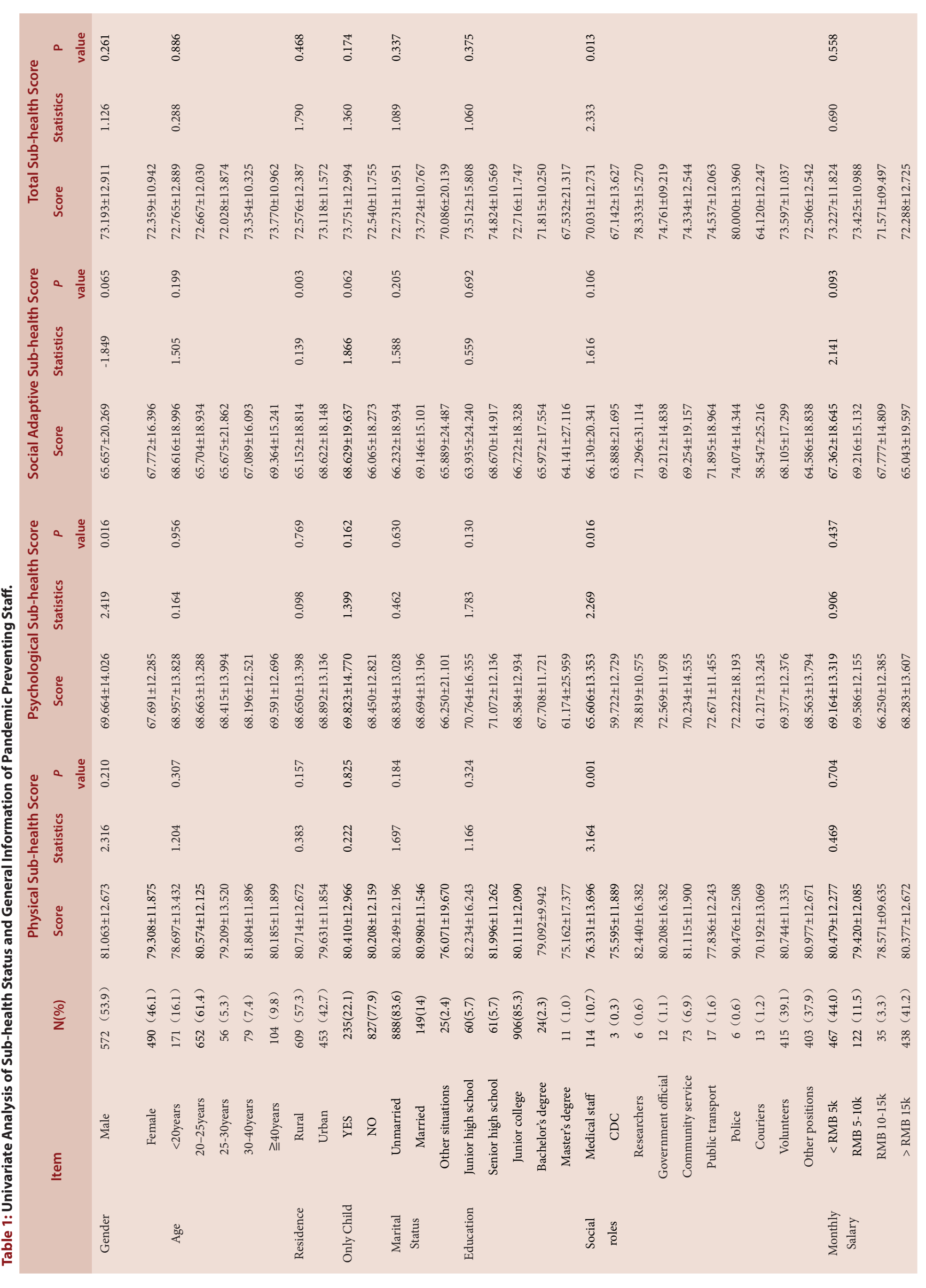


for total sub-health scores, there were differences across social roles $(P<0.05)$. (Table 2)

\section{Multivariate Analysis of Sub-health Status of People with Different Social Roles during the COVID-19 Pandemic}

A multivariate analysis $\left(\alpha_{\text {entry }}=0.05, \alpha_{\text {removal }}=0.1\right)$ was performed with physical sub-health status scores, psychological sub-health status scores, social adaptive sub-health status scores and total sub-health scores as dependent variables, whereas statistically significant variables from the univariate analysis were taken as independent variables (Table 3 ). The results showed that both social roles were the influencing factors for the physical sub-health status score $(P<0.05)$; gender was influencing factors for psychological sub-health status score $(P<0.05)$; (Table 4$)$

\section{DISCUSSION}

\section{Prevalence of SHS among People with Different Social Roles during the COVID-19 Pandemic}

A total of 1062 pandemic prevention and control staff were included; results showed that $62.1 \%$ of this population had SHS, which was consistent with the study findings of $\mathrm{Hu}$ Xianming et al. ${ }^{7}$ During the COVID-19 pandemic, the prevalence of SHS among pandemic preventing staff remained high, below the expectations for the general health condition. Among the three sub-health dimensions, the highest score was noted in physical sub-health status, followed by psychological sub-health status and social adaptive sub-health status. In the event of the COVID-19 pandemic, non-professionals may be worried about being infected with the virus due to a lack of medical knowledge, thereby resulting in a series of psychological stress reactions. Their psychological sub-health was characterized by a group of changes in terms of emotion, cognition and behaviors, including fear, anxiety, helplessness, frustration, etc; ${ }^{15}$ social adaptive sub-health manifested itself as inadaptability to the environment, troubled interpersonal relationships and dysfunctional roles. ${ }^{16}$ Low social adaptive sub-health status scores may be related to tough control measures as well as the universal impact on daily lifestyles, interpersonal communication and social roles. ${ }^{17}$

Table 2: Descriptive Statistics for Sub-health Status of Pandemic Preventing Staff $(n=1062)$.

\begin{tabular}{|ccccc|}
\hline Factor & $\begin{array}{c}\text { Full } \\
\text { Score }\end{array}$ & $\begin{array}{c}\text { Number } \\
\text { of Items }\end{array}$ & Score $(x \pm s)$ & $\begin{array}{c}\text { Mean Score by } \\
\text { Item }\end{array}$ \\
$\begin{array}{c}\text { Physical Sub- } \\
\text { health }\end{array}$ & 70 & 14 & $80.252 \pm 12.336$ & $5.732 \pm 0.881$ \\
$\begin{array}{c}\text { Psychological } \\
\text { Sub-health }\end{array}$ & 60 & 12 & $68.753 \pm 13.281$ & $5.729 \pm 1.106$ \\
$\begin{array}{c}\text { Social adaptive } \\
\text { Sub-health }\end{array}$ & 45 & 9 & $66.632 \pm 18.604$ & $7.403 \pm 2.067$ \\
$\begin{array}{c}\text { Total Score } \\
\text { Inthen }\end{array}$ & 175 & 35 & $72.808 \pm 12.034$ & $2.080 \pm 0.344$ \\
\hline
\end{tabular}

Table 3: Assignment of Independent Variables.

\begin{tabular}{|c|c|}
\hline $\begin{array}{l}\text { Independent } \\
\text { Variable }\end{array}$ & Value Assignment \\
\hline Gender & Male $=1 ;$ female $=2$ \\
\hline Social roles & $\begin{array}{c}\text { Medical staff }=1 ; \text { CDC }=2 \text {; scientific researchers }=3 ; \\
\text { Government officials }=4 ; \text { community service workers }= \\
5 ; \text { public transport personnel }=6 \text {; police }=7 \text {; couriers }=8 ; \\
\text { volunteers }=9 ; \text { staff in other positions }=10 ;\end{array}$ \\
\hline
\end{tabular}

Therefore, during the COVID-19 pandemic, they need more psychological care and material support. Psychological support facilities are expected to provide them with psychological services such as psychological counseling and psychological intervention. Only the assurance of good health can guarantee them to perform well at work, ${ }^{18}$ thus ensuring proper implementation of COVID-19 prevention and control.

\section{Relationship between Place of Residence and Social Adaptive SHS}

This study demonstrated that statistically significant social adaptive sub-health status scores were observed in places of residence from the univariate analysis $(P<0.05)$, while the results of the multivariate analysis were not statistically significant $(P>0.05)$. However, the prevalence of social adaptive SHS was higher in urban areas than in rural areas. The reasons may include limited space of activities and lifestyle changes resulted from indoor isolation of people living in cities during the pandemic. In contrast, the living environment in rural areas is relatively comfortable and has less restriction, leaving few changes from the prepandemiclifestyle. ${ }^{19}$ Dysfunctional roles developed as social adaptive sub-health. Due to the impact of the COVID-19 pandemic, many enterprises suspended production and even forced to shut down. Urban wage earners were unable to return to work as scheduled, which caused most people to develop the incapability to adapt due to the interruption of social function. On the contrary, rural residents found more freedom to arrange their work and acquired fewer influences on lifestyles, thereby causing no significant inadaptability. ${ }^{16}$

\section{Relationship between Social Roles and Physiological Sub-health Status}

This study showed that couriers, medical staff and CDC personnel had lower physical sub-health status scores, all of whom were at physiological SHS. About the reasons for low physical sub-health status scores for couriers, we speculated that couriers were responsible for delivering necessary supplies to people quarantined indoors in response to inconvenience caused by COVID-19. These employees continued working under heavy load and increased risk of exposure to COVID-19 without adequate rest when they had too much work stress to handle. Low physical sub-health status scores observed for CDC personnel and medical staff may be related to engagement during which they were responsible for epidemiological investigations, nucleic acid tests and detection of virus specimens. Furthermore, medical staff needed

\begin{tabular}{|c|c|c|c|c|c|}
\hline Variable & Bvalue & $\begin{array}{l}\text { Standard } \\
\text { Error }\end{array}$ & Bvalue & Tvalue & Pvalue \\
\hline \multicolumn{6}{|l|}{$\begin{array}{c}\text { Physical Sub health } \\
\text { Score }\end{array}$} \\
\hline Constant Item & 76.935 & 1.088 & - & 70.721 & $<0.001$ \\
\hline Social roles & 0.375 & 0.115 & 0.099 & 3.251 & 0.001 \\
\hline \multicolumn{6}{|l|}{$\begin{array}{l}\text { Psychological Sub- } \\
\text { health Score }\end{array}$} \\
\hline Constant Item & 71.637 & 1.259 & - & 56.878 & $<0.001$ \\
\hline Gender & -1.973 & 0.816 & -0.074 & -2.419 & 0.016 \\
\hline
\end{tabular}

${ }^{1)} R^{2}=0.010$, 调整 $R^{2}=0.009, F=10.569, \quad P=0.001 ; \quad{ }^{2)} R^{2}=0.005$, 调整

$R^{2}=0.005, \quad F=5.850, P=0.016$ 
to carry out important tasks such as managing the infected cases, putting them at a greater risk of viral infection and under tremendous pressure, both physically and psychologically. It is reported that SHS can be prevented from progressing to disease by providing effective health guidance, healthcare interventions, protection knowledge and protective skills. ${ }^{20}$ Therefore, we need to pay more attention to the physical and mental health of these groups of people by taking into consideration of health consultation, protection guidance, health examination and medical security; managers are recommended to adjust human resource allocation on time and to optimize human resource management, ${ }^{21}$ allowing relevant staff to have adequate rests for recovery of physical and mental health, to avoid the progression into illness where possible. In this way, these staff will always be well prepared to provide their services as their physical and mental health is maintained.

\section{Relationship between Genders and Psychological Sub- health Status}

For psychological dimension, statistically, significant differences were found between men and women who were assessed as $69.664 \pm 14.026$ and $67.691 \pm 12.285$ respectively $(P=0.016)$, which meant women had slightly worse mental health status compared with men and both were in SHS. This result was consistent with the study findings of Yang Yang et al. ${ }^{22,23}$ and Zhang Yingzhi et al. ${ }^{22,23}$ However, this difference may be driven by the large sample size, which should be treated with caution. During the COVID-19 pandemic, frequent contacts with different groups of people in the society increased the risk of infection for the pandemic preventing staff, result in higher mental stress to them. Wang Xiu et al. ${ }^{24}$ believed that SHS in women were mainly characterized by psychological aspects and that women were more likely to develop anxiety, tension, depression as well as other emotional disorders, resulting in psychological disorders since they tended to be emotional. Due to the COVID-19 pandemic, women were more prone to various psychological reactions such as anxiety and tension. Shi Ling et al. ${ }^{25}$ believed that these abnormal psychological reactions would further affect their physiological function, coupled with different individual constitutions, work environment, family conditions, social environment and some stressful life events, all of which may lead to adverse effects on physical and mental health in women. In addition, prolonged duration of work, increased stress and higher work intensity during the pandemic had also broken the work-family balance; ${ }^{26}$ women typically served more than one role by working and taking care of their family with inadequate time for self-regulation. The emotional support given by family members has been shown to help resolve the work-family conflict, ${ }^{27}$ thus family support and understanding are very important. As indicated by this questionnaire survey, women had lower psychological sub-health status scores, for which management staff is recommended to take various measures to provide women with more emotional care and psychological support. Also, additional support is encouraged with regard to female staff, including material supplies to their families, assistance in learning for their children and other necessary support for their daily life. These will serve as a strong conviction for women to throw themselves into the battlegrounds free of family concerns and to help recover from their SHS.

\section{Limitations of the Study}

First, although much effort has been spent to mobilize a variety of occupational groups to participate in this survey, biased occupational distribution still existed in the population. Second, there was also an age bias for the study population between 18 to 40 age group young adults and a small number of people aged over 50 years, which may be related to the rare use of electronic products by the elderly. Third, the data sources of this study were mostly limited to Guangdong Province, China, which brought regional limitations to the study findings along with the publication bias. This survey is expected to cover other regions or populations for further validation in the future.

\section{CONCLUSION}

Currently, COVID-19 has been declared as a pandemic and the "draconic" measures taken by China for disease control have been proven effective. However, travel bans make a significant impact on the daily lives of people. Employees from some industries must continue working and provide services to maintain the essential operations of the society. In these individuals, the prevalence of SHS is high due to heavier workload and increased risk of virus exposure. Among them, the medical staff, CDC personnel and couriers need assistance in maintaining their physical health. It is recommended that managers make prompt decisions to optimize human resources management as well as providing health consultations, adequate rests and medical security. Since women need more mental health support, goods and necessary supports for a daily living may be provided to maintain their work-family balance. These ordinary workers play an important part in COVID-19 prevention and control in China. Only by maintaining the good health of those fighting against COVID-19 can we stop the spread of the virus more effectively.

\section{ACKNOWLEDGEMENT}

The author is very grateful to the workers who participated in this study and their efforts.

\section{CONFLICT OF INTEREST}

The authors have no conflicting interest.

\section{ABBREVIATIONS}

COVID-19: The Coronavirus Disease 2019; SHS: The Sub-Health Status; WHO: World Health Organization; CDC: Center for disease control and prevention.

\section{REFERENCES}

1. Cheng L, Xie JG. Interpretation of COVID-19 diagnosis and treatment scheme (trial seventh edition). The Medicine Herald. 2020;1-6.

2. Peng YN, Wu XH, Shen YJ, Chen Bo, Zhuang J. Practice of medical social workers in COVID-19 prevention and control under the theory of social role $[\mathrm{J}]$. China Social Work. 2020;(09):12-6.

3. Zhao RQ, Song ZF. Research progress of sub-health problems. Chinese Journal of Social Medicine. 2002;19(1):10-3.

4. Lee J, Dong SO, Lee Y, Kim SH, Lee S. Recognition of and interventions for Mibyeong (subhealth) in South Korea: A national web-based survey of Korean medicine practitioners. Integr Med Res. 2014;3(2):60-6.

5. Brown RJ. Introduction to the special issue on medically unexplained symptoms: Background and future directions. Clinical Psychology Review. 2007;27(7):769-80.

6. Fu SL. A new perspective on health in the $21^{\text {st }}$ century. Shanghai: Shanghai Science and Technology Education Press. 2000;1-20.

7. Hu XM, Lu SQ, Bai LX, Wang LX, Guo XY, Wang Q, et al. Analysis on the subhealth status of some population in taiyuan city and its influencing factors of social psychology. Health Education in China. 2006;(11):807-10.

8. Lan SQ, Mai WJ, Liang MF, Huang RL. Investigation and analysis of sub-health status of nurses in primary hospitals in foshan city and countermeasures. General Nursing. 2012;10(9):844-5

9. Sun XM, Huang SH. Epidemiological investigation of subhealth status of 600 Hong Kong population. Journal of Tropical Medicine. 2012;12(1):83-6.

10. Yuan $P$, Tang YL, Yu XF. Analysis on the sub-health status of jiangmen residents and its influencing factors. Public Health in China. 2012;28(6):844-7.

11. Meng SJ, Yan YX, Liu YQ, Dong J, Song MS, Wang W. Study on the sub-health status of medical workers and its influencing factors. Chinese General Practice. 2013;6(1):61-4.

12. Chen JY, Cheng JR, LiuYY, Tang Y, Sun XM, Wang T, et al. Associations between breakfast eating habits and health-promoting lifestyle, suboptimal health 
status in Southern China: A population based, cross sectional study. Journal of Translational Medicine. 2014;12(1).

13. Xu J, Feng LY, Luo R, Qiu JC, Zhang JH, Zhao XS, et al. Study on reliability and validity of subhealth rating scale. Journal of Southern Medical University. 2011;31(1):33-8.

14. Chen WPWH, Frederick GH. A novel coronavirus outbreak of global health concern. Lancet. 2020;395(10223):30185-9.

15. He Q, Wang BL, Mo CN, Tian XQ, Zhang N, Zhang MM, et al. The impact of earthquake disaster on the mental health of military medical personnel in emergency rescue. Chinese Journal of Practical Nursing. 2018;34(17):1314-6.

16. Deng H, Shi HL, Fei B, Wang PWZL. Review on the research status of subhealth in China. Journal of Mudanjiang Normal University. 2009;(4):33-6.

17. Xu MC, Zhang Y. Investigation on the psychological status of the first clinical first-line support nurses against novel coronavirus pneumonia. Nursing Research. 2020;3(34):368-70.

18. Kong CX, Lin J, Zhou XD. The influence of health status of nursing staff on the quality of disinfection supply room. Modern Preventive Medicine. 2012;39(13):3309-10.

19. Shi P, Liu Z, Zhang YB, Wang J, Hui BN, Wang T. Analysis of anxiety status and influencing factors in tumor patients with COVID-19 epidemic. Modern Oncology. 2020;8(9):1-3.
20. Cai WZ. Investigation on the current situation of occupational injury of medical personnel and analysis of related influencing factors. 2009;1-175.

21. Huang MY, Lin HJ, Wang J. Effects of optimizing human resource utilization on job burnout and mental health status of pediatric nursing staff. Journal of Chinese Medicine Management. 2017;25(3):87-9.

22. Zhang YZ. The cause analysis and countermeasure of nurses 'sub-health Medical Theory and Practice. 2005;(2):229-30.

23. Yang Y. A study on the relationship between sub-health status survey and physical exercise of medical staff in x city. 2017;1-63.

24. Wang X, HeYM. Analysis on the difference of subhealthy population of different genders in China. Public Health in China. 2012;28(1):15-6.

25. Shi L. Clinical study on the distribution of symptoms and quality of life in menopausal women. 2007;1-55

26. Zurlo MC, Vallone F, Smith AP. Work-family conflict and psychophysical health conditions of nurses: Gender differences and moderating variables. Jpn J Nurs Sci. 2020;e12324. DOI:10.1111/jjns.12324

27. Zhang YH, Tang L, Jiang Q, Zhou CF, Lin WFW, et al. Emergency off-site rescue to combat COVID-19 nurses early psychological distress investigation. Journal of Nursing. 2020;35(5):1-4.

Cite this article: Chen Z, Liu Q, Lu W, Pan Y, Shan M. The Sub-health Status of Different Social Roles and the Analysis of its Influencing Factors during the 2019 Coronavirus Disease Pandemic. Int J Med Public Health. 2020;10(3):135-40. 